\title{
Snip-Tip Dispensing Plastic Container Closure
}

National Cancer Institute

\section{Source}

National Cancer Institute. Snip-Tip Dispensing Plastic Container Closure. NCI Thesaurus.

Code C96134.

Conical closure that is turned onto a container. The tip is cut off to open the container. 